\title{
TU/e emonownen

\section{Towards data-driven LPV controller synthesis based on frequency response functions}

\section{Citation for published version (APA):}

Bloemers, T., Tóth, R., \& Oomen, T. (2019). Towards data-driven LPV controller synthesis based on frequency response functions. In IEEE 58th Conference on Decision and Control (pp. 5680-5685). [9029958] (Proceedings of the IEEE Conference on Decision and Control; Vol. 2019-December). Institute of Electrical and Electronics Engineers. https://doi.org/10.1109/CDC40024.2019.9029958

\section{Document license:}

CC BY-NC-ND

DOI:

10.1109/CDC40024.2019.9029958

Document status and date:

Published: 01/12/2019

\section{Document Version:}

Accepted manuscript including changes made at the peer-review stage

\section{Please check the document version of this publication:}

- A submitted manuscript is the version of the article upon submission and before peer-review. There can be important differences between the submitted version and the official published version of record. People interested in the research are advised to contact the author for the final version of the publication, or visit the $\mathrm{DOI}$ to the publisher's website.

- The final author version and the galley proof are versions of the publication after peer review.

- The final published version features the final layout of the paper including the volume, issue and page numbers.

Link to publication

\section{General rights}

Copyright and moral rights for the publications made accessible in the public portal are retained by the authors and/or other copyright owners and it is a condition of accessing publications that users recognise and abide by the legal requirements associated with these rights.

- Users may download and print one copy of any publication from the public portal for the purpose of private study or research.

- You may not further distribute the material or use it for any profit-making activity or commercial gain

- You may freely distribute the URL identifying the publication in the public portal.

If the publication is distributed under the terms of Article 25fa of the Dutch Copyright Act, indicated by the "Taverne" license above, please follow below link for the End User Agreement:

www.tue.nl/taverne

Take down policy

If you believe that this document breaches copyright please contact us at:

openaccess@tue.nl

providing details and we will investigate your claim. 


\title{
Towards Data-Driven LPV Controller Synthesis Based on Frequency Response Functions
}

\author{
Tom Bloemers, Roland Tóth and Tom Oomen
}

\begin{abstract}
Synthesizing Linear Time-Invariant (LTI) controllers directly from Frequency Response Function (FRF) measurements is at the core of many successful industrial applications. However, increasing performance expectations necessitate extending these approaches for multiple operating points, viewing the plant as a Linear Parameter-Varying system. The aim of this paper is to develop FRF data based controller synthesis to a larger class of systems by leveraging on recent developments in LPV system theory. The developed method is based on a global LPV controller parametrization with a finite impulse response structure which ensures local stability and performance of the closed-loop behavior by design. A case study confirms that the developed control design procedure, using only measurement data, can be effectively used to design LPV controllers resulting in stability and high performance of the closed-loop system on the specified region of operating conditions in comparison to LTI controllers.
\end{abstract}

\section{INTRODUCTION}

Frequency response function (FRF) based controller synthesis enables direct design of controllers based on nonparametric models and provides an automated alternative to classical controller tuning approaches, including Nyquist designs [1]. FRF measurements have traditionally been used as a basis for manual tuning of Proportional-Integral-Derivative (PID) controllers through loop-shaping, relying on graphical tools such as a Bode diagram or Nyquist plot. This has been further developed towards data-driven approaches, using FRF measurements as a basis, to synthesize linear time-invariant (LTI) controllers. First these methods were developed for PID controllers [2], [3]. More recently, developments have been made towards more general control structures, with the main focus on robust control [4]-[7]. By these approaches, a controller is designed such that performance and stability specifications for a given set of FRF measurements are achieved. From an identification perspective, this provides an alternative to control-oriented identification followed by model based controller synthesis [8].

Many systems encountered in practice exhibit nonlinear (NL) behavior. Common control design methods for such systems aim at the design of an LTI controller at an operating point, such that locally sufficient robustness in terms of performance and stability are achieved. In practice,

T. Bloemers and R. Tóth are with the Eindhoven University of Technology, Department of Electrical Engineering, Control Systems Group, Eindhoven, The Netherlands. T. Oomen is with the Eindhoven University of Technology, Department of Mechanical Engineering, Control Systems Technology Group, Eindhoven, The Netherlands. Email: t.a.h.bloemersatue.nl

This work has received funding from the European Research Council (ERC) under the European Union's Horizon 2020 research and innovation programme (grant agreement nr. 714663). these controllers are often designed on the basis of an FRF measurement of the system, obtained at the operating point, through either manual loop-shaping or through more advanced model based control synthesis techniques such as $\mathcal{H}_{2}, \mathcal{H}_{\infty}$ or $\mu$-synthesis [9], [10]. However, these LTI based methods can be conservative, resulting in a significant sacrifice of performance as they try to deal with the nonlinear dynamics through robustness. As an alternative, the framework of linear parameter-varying (LPV) systems has been introduced to tackle the control of nonlinear systems through controller parametrizations that vary the control law with the operating point changes of the system and relying on extensions of well established LTI methods, with many successful applications such as [11]-[13].

In LPV systems, the input-output (IO) map is considered to be linear as in the LTI framework, but this IO map is assumed to be a function of a so-called scheduling variable $p$, capturing, e.g., the operating point dependent dynamics. The class of LPV systems is supported by well-developed identification theory and control design methodologies. LPV control approaches extend common LTI methods, e.g., $\mathcal{H}_{\infty^{-}}$ control [14]-[16], providing either local or global stability and performance guarantees. When synthesizing an LPV controller for a nonlinear plant, performance specifications are often enforced through shaping methods in the frequency domain. In the identification literature, recent developments have been made towards nonparametric modeling through FRF identification of LPV systems. These developments follow the paths of local identification in [17], [18] and more recently also a global approach to FRF identification of LPV systems has been developed [19]. For an overview of FRF representations of LPV systems see [20].

Although data-driven controller synthesis based on FRF measurements enables a systematic design approaches within the LTI framework, at present these methods are limited when applied to LPV systems. First steps towards datadriven LPV controller synthesis in the frequency domain are presented in [21]. Here a gain-scheduled controller is sought through an optimization problem, where local performance and stability requirements are characterized in terms of lower bounds on the bandwidth and both gain and phase margins. In [22] a gain-scheduled controller is synthesized through constraints in the Nyquist diagram, where a manual design of the open-loop transfer is required to guarantee local stability.

Inspired by these methods, the main contribution of this paper is a synthesis procedure for data-driven LPV controller design based on local, so-called "frozen" FRF measurements. The presented methodology can be thought of as an improved 
version of [21], [22], in the sense that the desired control objectives are formulated in terms of a model matching problem, where control objectives are posed in terms of frequency domain shaping filters. Through a set of basis functions, a global parametrization of the dependency of the controller on the operating points is specified, for which guarantees on stability and performance are provided in the local sense. The solution to the problem is obtained via convex optimization. The capabilities of the approach are demonstrated on a simulation example of a nonlinear massspring-damper (MSD) system with a duffing spring.

The paper is organized as follows: in Section II, the class of considered models and controllers are defined and the problem setting is formulated. In Section III, the proposed stability and performance criteria are stated and a convex optimization approach to synthesize LPV FIR controllers is derived. Section IV demonstrates the proposed methodology, through a simulation example, by designing a fixed-order LPV FIR controller for a nonlinear system. Finally, conclusions are drawn in Section V.

Notation: $\mathbb{R}$ denotes the set of real numbers. For a given vector $x \in \mathbb{R}^{n_{\mathrm{x}}}, x_{j}$ denotes the $j$-th component of $x$. The imaginary unit is denoted by $i=\sqrt{-1}$ and $\Re\{\cdot\}$ and $\Im\{\cdot\}$ represent the real and imaginary part of a complex variable respectively. The set of real rational proper and stable transfer functions is denoted by $\mathcal{R} \mathcal{H}_{\infty}$.

\section{PROBLEM FORMULATION}

Given a single-input single-output (SISO) nonlinear dynamical system in continuous-time:

$$
F:\left\{\begin{array}{l}
\dot{x}=f(x, u) \\
y=h(x, u)
\end{array}\right.
$$

for which we would like to design a gain-scheduled LPV controller based on data without actually identifying or modeling the underlying dynamics. In practice, it is often possible to gather measurement data from systems like (1). Hence, consider a set of local linear models of (1) at the operating points ${ }^{1}\left(x_{0}, u_{0}\right) \in \mathbb{X}_{0} \times \mathbb{U}_{0}$, which can be thought of as discrete-time linearizations of (1) at these points [23], [24]. For the sake of simplicity here we assume that there is no noise or unmeasured disturbances perturbing the data collection. Furthermore, we consider that $\mathbb{X}_{0} \subset \mathbb{X}$ and $\mathbb{U}_{0} \subset \mathbb{U}$, where $x: \mathbb{R} \rightarrow \mathbb{X} \subseteq \mathbb{R}^{n_{\mathrm{x}}}$ is the state variable, $u: \mathbb{R} \rightarrow \mathbb{U} \subseteq \mathbb{R}^{n_{\mathrm{u}}}$ and $y: \mathbb{R} \rightarrow \mathbb{Y} \subseteq \mathbb{R}^{n_{\mathrm{y}}}$ are the input and output signals of (1), respectively. These discrete-time linear models are denoted $G_{p}\left(e^{i \omega}\right)$, dependent on a so-called scheduling variable $p=\mu\left(x_{0}, u_{0}\right)$, which expresses the operating condition in terms of the scheduling map $\mu\left(x_{0}, u_{0}\right)$. Assume that $G_{p}\left(e^{i \omega}\right)$ are stable and their FRF measurements are available for a given set of operating points $\left\{p^{(\tau)}\right\}_{\tau=1}^{N_{\mathrm{p}}} \in \mathcal{P} \subset \mathbb{P}=\mu\left(\mathbb{X}_{0}, \mathbb{U}_{0}\right)$ and a set of discrete frequencies $\omega \in \Omega \subset[0, \pi]$.

Controller design for (1) is considered in terms of the interconnection in Figure 1 and the performance

\footnotetext{
${ }^{1}\left(x_{0}, u_{0}\right)$ is an operating point of (1) if $0=f\left(x_{0}, u_{0}\right)$.
}

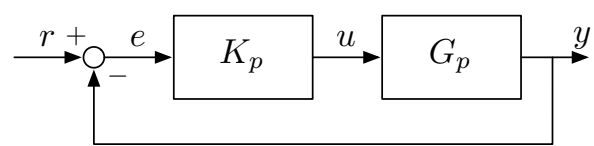

Fig. 1. LPV representation of the closed-loop interconnection. $G_{p}$ is a linear system corresponding to the local frozen behavior of $F$ at the operating point $p$ and $K_{p}$ is an LPV controller scheduled by $p$.

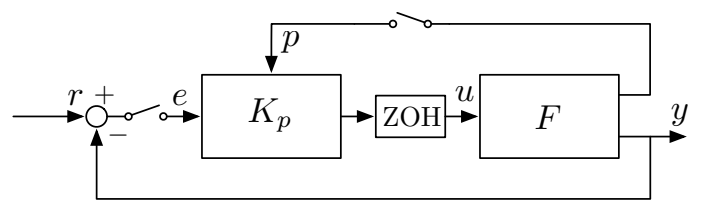

Fig. 2. Interpretation of the closed-loop implementations of the LPV controller with the plant. Here $K_{p}$ is the discrete-time LPV controller scheduled by a time-varying $p$, whereas $F$ represents the continuous-time nonlinear system (1) from which $p$ is measured.

specifications are expressed by a model matching filter $M \in \mathcal{R} \mathcal{H}_{\infty}$. Here, the objective is to design a controller that depends on the scheduling variable, such that (a) for all operating conditions the closed-loop system is stable in the local sense and (b) for all operating conditions, the error between the set of closed-loop systems and the model matching filter is below an acceptable threshold. More specifically, the goal is to design a controller $K_{p}$ dependent on $p$ such that

(a) $\forall p \in \mathbb{P}$ the closed-loop transfer function in Figure 1

$$
T_{p}\left(e^{i \omega}\right)=\frac{L_{p}\left(e^{i \omega}\right)}{1+L_{p}\left(e^{i \omega}\right)},
$$

with $L_{p}\left(e^{i \omega}\right)=G_{p}\left(e^{i \omega}\right) K_{p}\left(e^{i \omega}\right)$, is stable, i.e., the poles of the transfer function $T_{p}$ lie within the unit circle; (b)

$$
\sup _{\omega \in \Omega}\left|T_{p}\left(e^{i \omega}\right)-M\left(e^{i \omega}\right)\right|<\gamma,
$$

is below a given threshold $\gamma \geq 0, \forall p \in \mathbb{P}$.

While (a) describes the natural local stability expectation, (b) expresses the performance objective. The resulting controller $K_{p}$ here is more than just a robust design because its parameters depend on $p$ and can be thought of as a data-driven gain-scheduled controller. During the controller design $p$ is considered to be constant (see Figure 1), but during implementation $p$ is time-varying. The controller implementation is illustrated in Figure 2. Naturally, the question then arises: how to synthesize such a controller with respect to a sampled data structure $\mathcal{P}$ and $\Omega$.

\section{DATA-DRIVEN LPV CONTROLLER SYNTHESIS}

In practice, when a model of (1) is not known, only (i) measurements of (1) at a finite set of points $\mathcal{P}$ and at only frequencies $\Omega$ are available. Hence, requirements (a) and (b) can only be satisfied locally at constant operating conditions $p \in \mathcal{P}$. 


\section{A. Controller parametrization}

The controller parametrization is first introduced in the frequency domain, whereas later on when the the implementation aspects are discussed, a time domain interpretation is given. The controller is parametrized as a discrete-time fixed $n_{\mathrm{b}}$-order FIR structure

$$
K_{p}\left(e^{i \omega}\right)=\sum_{\ell=0}^{n_{\mathrm{b}}} b_{\ell}(p) e^{-i \omega \ell}
$$

with the frequency independent coefficients $b_{\ell}(p)$ being parametrized with a priori chosen set of basis functions $\left\{\phi_{j}^{\ell}(p)\right\}_{j=1}^{n}$ as

$$
b_{\ell}(p)=\sum_{j=1}^{n} c_{j}^{\ell} \phi_{j}^{\ell}(p) .
$$

The set $\left\{\phi_{j}(p)\right\}_{j=1}^{n}$ can be chosen as affine, polynomial, rational, or piecewise linear basis functions in $p$, defining the function class to which the scheduling dependence of the controller is restricted to. We denote by $\theta=\left[\begin{array}{lllllll}c_{1}^{0} & \ldots & c_{1}^{n_{b}} & \ldots & c_{n}^{0} & \ldots & c_{n}^{n_{b}}\end{array}\right]$ the controller coefficients.

\section{B. Performance}

The closed-loop performance requirements are defined in terms of an ideal transfer function $M\left(e^{i \omega}\right)$. To measure the satisfaction of the shaped local control behavior with respect to $M\left(e^{i \omega}\right)$, a weighted $\mathcal{H}_{\infty}$-norm is considered in terms of

$$
\begin{array}{ll}
\min _{\theta} & \gamma \\
\text { s.t. } & \left|W\left(e^{i \omega}\right)\left(T_{p}\left(e^{i \omega}, \theta\right)-M\left(e^{i \omega}\right)\right)\right|<\gamma \\
& \forall \omega \in \Omega \\
& \forall p \in \mathcal{P}
\end{array}
$$

where $W\left(e^{i \omega}\right)$ is an additional weighting filter used to specify the relative importance over the range of frequencies. This is in line with typical control design methodologies including design requirement (b). Without a parametric form of the model, optimization problem (6) is nonconvex in the design variable $\theta$, and can therefore be difficult to solve. A linearizing transformation is performed through multiplication by $1+L_{p}\left(e^{i \omega}, \theta\right)$. This results in the model matching problem

$$
\begin{array}{ll}
\min _{\theta} & \gamma \\
\text { s.t. } & \left|W\left(e^{i \omega}\right)\left(\left(1-M\left(e^{i \omega}\right)\right) L_{p}\left(e^{i \omega}, \theta\right)-M\left(e^{i \omega}\right)\right)\right|<\gamma \\
& \forall \omega \in \Omega \\
& \forall p \in \mathcal{P}
\end{array}
$$

which is a second order cone optimization problem that is convex in $\theta$. Note that in general, the optimization problem posed in (7) is not equivalent to (6). If there exists a $\theta$ such that $T_{p}\left(e^{i \omega}, \theta\right)=M\left(e^{i \omega}\right) \forall p \in \mathcal{P}$, then (7) and (6) are equivalent. However, if such a $\theta$ does not exist, then the optimal solution $\theta^{\star}$ of (7) represents the optimal solution of (6) only if the weighting filter in (6) is substituted with $W\left(e^{i \omega}\right)\left(1+L_{p}\left(e^{i \omega}, \theta^{\star}\right)\right)$.

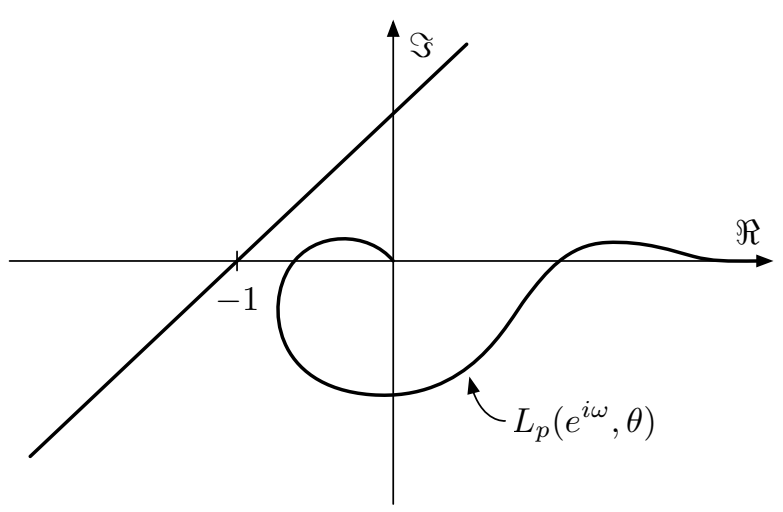

Fig. 3. Graphical interpretation of the simplified Nyquist stability criterion. The line restricts the Nyquist curve to lie to the right of the critical point $(-1,0 i)$. The Nyquist curve $L_{p}\left(e^{i \omega}, \theta\right)$ is shown for a constant operating condition $p$ and fixed $\theta$.

\section{Stability}

To ensure local stability of the closed-loop, a Nyquist constraint is introduced, which will amount to requirement (a). To this end, the Nyquist theorem is presented first, after which a simplified version is given. Next, a set of constraints are derived, which guarantee local stability of the closed-loop system in Figure 1.

Let $\mathcal{C}$ denote the closed contour that encircles the exterior of the unit disc, i.e., the area of instability. Furthermore, we denote by $P$ the number of unstable poles of $L_{p}\left(e^{i \omega}, \theta\right), Z$ is the number of zeros encircled by $\mathcal{C}$, i.e., the number of unstable closed-loop poles of $T_{p}\left(e^{i \omega}, \theta\right)$ and let $N$ denote the number of clockwise encirclements of $L_{p}\left(e^{i \omega}, \theta\right)$ around the point $(-1,0 i)$.

Theorem 1 (Nyquist stability criterion [25]): The closedloop $T_{p}\left(e^{i \omega}, \theta\right)$ is stable, if and only if $L_{p}\left(e^{i \omega}, \theta\right)$ makes $N=$ $Z-P$ clockwise encirclements around the point $(-1,0 i)$, and $L_{p}\left(e^{i \omega}, \theta\right)$ does not pass through the point $(-1,0 i)$.

The proof is based on Cauchy's argument principle and the reader is referred to [25] for the proof. In case the plant and controller are stable, i.e., $P=0$, then the following simplified Nyquist criterion holds

Corollary 1 (Simplified Nyquist theorem): Assume that $L_{p}\left(e^{i \omega}, \theta\right)$ has no unstable poles. Then the closed-loop system $T_{p}\left(e^{i \omega}, \theta\right)$ is stable if and only if the Nyquist contour does not encircle nor cross the point $(-1,0 i)$.

Proof: The proof follows from Theorem 1 by taking $Z=P=0$.

Under the condition that $G_{p}\left(e^{i \omega}\right)$ is stable with the choice of an FIR controller structure, Corollary 1 is exactly the condition required for local stability. In order to ensure that the Nyquist contour does not encircle nor cross the origin, the Nyquist curve evaluated for $\omega \geq 0$ is constrained to lie below a line going through the origin. This is graphically displayed in Figure 3. This results in the following corollary.

Corollary 2: Suppose that $L_{p}\left(e^{i \omega}, \theta\right)$ has no unstable poles and that the image of $L_{p}\left(e^{i \omega}, \theta\right)$ lies below the line

$$
z=x+i \alpha(x+1)
$$


in the complex plane, with $x \in \mathbb{R}$ and $\alpha>0 \in \mathbb{R}$. Then the closed-loop system $T_{p}\left(e^{i \omega}, \theta\right)$ is stable and $L_{p}\left(e^{i \omega}, \theta\right)$ satisfies that

$$
\Im\left\{L_{p}\left(e^{i \omega}, \theta\right)\right\}<\alpha\left(\Re\left\{L_{p}\left(e^{i \omega}, \theta\right)\right\}+1\right) .
$$

This corollary states that the Nyquist curve lies below a line in the complex plane with slope $\alpha$ and provides a sufficient condition for the closed-loop in Figure 1 to be locally stable for each operating point $p$. Note that these stability concepts give no guarantees for stability of the closed-loop behavior in Figure 2 for varying $p$. For that the available information in the frozen FRFs is insufficient and concepts like the global FRF forms presented in [19], [20] might provide stability concepts in the future, but currently theoretical tools are missing for global stability analysis purely on frequency domain data. However, if the scheduling variation is sufficiently slow, stability can be guaranteed in the global sense based on purely local conditions as motivated in [23], however there is no exact characterization of how slow that scheduling variation needs to be.

\section{Fixed-order LPV controller synthesis}

By combining the conditions for performance (7) and stability (9) introduced in the previous subsections, a convex optimization problem for data-driven synthesis of fixed-order LPV FIR controllers is formulated

$$
\begin{array}{ll}
\min _{\theta} & \gamma \\
\text { s.t. } & \left|W\left(e^{i \omega}\right)\left(\left(1-M\left(e^{i \omega}\right)\right) L_{p}\left(e^{i \omega}, \theta\right)-M\left(e^{i \omega}\right)\right)\right|<\gamma \\
& \Im\left\{L_{p}\left(e^{i \omega}, \theta\right)\right\}<\alpha\left(\Re\left\{L_{p}\left(e^{i \omega}, \theta\right)\right\}+1\right) \\
& \forall \omega \in \Omega \\
& \forall p \in \mathcal{P} .
\end{array}
$$

The optimization problem (10) is convex in $\theta$, due to the FIR structure (4) and linear parametrization (5), but has an infinite number of constraints. A practical solution to solve (10) is to sample the frequency space $\Omega=\left\{\omega^{k}\right\}_{k=1}^{N_{\omega}}$ scheduling space $\mathcal{P}=\left\{p^{(\tau)}\right\}_{\tau=1}^{N_{\mathrm{p}}}$ which is in line with the availability of such data in practice. Then, (10) is solved as a second-order cone optimization problem. Likewise, (10) can also be posed as a set of linear matrix inequalities. It is important that $\Omega$ expresses all relevant frequencies, so the sampled condition (9) implies local stability [5]. Additionally, $\mathcal{P}$ can be chosen such that the sampled version of (3) actually implies the performance bound. Results to optimally determine such a set of operating points can be found in [26].

\section{E. Controller implementation}

While the controller is designed with fixed $p$, during implementation each $b_{\ell}$ is evaluated with $p$ obtained from the system at sample $k$, what we can denote as $b_{\ell}(p(k))$. Based on this, the controller is implemented according to the interconnection given in Figure 2, for which the signal relations are as follows

$$
u(k)=\sum_{\ell=0}^{n_{b}} b_{\ell}(p(k)) e(k-\ell) .
$$

As was discussed earlier, $b_{\ell}(p(k))$ is parametrized through basis functions $\left\{\phi_{j}(p)\right\}_{j=1}^{n}$. For example, $b_{\ell}$ can be chosen to have polynomial dependence on the scheduling variable $p$ :

$$
\left\{\phi_{j}(p)\right\}_{j=1}^{n}=\left\{1, p(k), p^{2}(k), \ldots, p^{n-1}(k)\right\} .
$$

With a polynomial dependence of order $n,(11)$ results in the following signal relations

$$
u(k)=\sum_{\ell=0}^{n_{b}}\left(c_{1}^{\ell}+c_{2}^{\ell} p(k)+\cdots+c_{n+1}^{\ell} p^{n}(k)\right) e(k-\ell) .
$$

\section{EXAMPLE}

Consider a 2nd-order mass-spring-damper system with nonlinear a spring, specifically a Duffing spring, obeying the continuous-time dynamical relations

$$
m \ddot{x}(t)+b \dot{x}(t)+k_{1} x(t)+k_{2} x^{3}(t)=F(t),
$$

where $x$ denotes the displacement of the mass $m$, $F(t) \in \mathbb{U}$ denotes the actuation force acting on the mass, $b$ is the damping coefficient and $k_{1}, k_{2}$ are the spring coefficients. Furthermore, we consider the operating range of the Duffing spring to be limited to $x(t) \in[-\sqrt{2}, \sqrt{2}]=\mathbb{X}$.

An LPV form of (14) is obtained by introducing the scheduling variable $p(t)=\mu(x(t))=m_{\mathrm{c}}+w_{\mathrm{c}} x^{2}(t)$ such that

$$
m \ddot{x}(t)+b \dot{x}(t)+k_{1} x(t)+k_{2} p(t) x(t)=F(t),
$$

with $p \in \mathbb{P}=[-1,1]$ and $x^{2} \in[0,2]$. Here $m_{\mathrm{c}}$ and $w_{\mathrm{c}}$ are used for centering and normalization, respectively. Furthermore, we assume that the system is actuated and sampled under a zero-order hold setting at a sampling time of $T_{s}=0.005$ [s]. FRF data, shown in Figure 4, is obtained at frozen operating points $p \equiv\left\{p^{(\tau)}\right\}_{\tau=1}^{N_{p}}=\mathcal{P}$, gridded on an equidistant regular grid $\mathcal{P}$ consisting of $N_{p}=11$ points and a frequency grid $\left\{\omega^{(k)}\right\}_{k=1}^{N_{\omega}}=\Omega$ consisting of $N_{\omega}=400$ logarithmically spaced frequency points in the range $\Omega \subset[0, \pi]$.

In terms of control specifications, we would like to achieve reference tracking according to Figure 1 with a rise time of 1 second and an overshoot below $5 \%$. This is expressed in terms of a model matching filter $M\left(e^{i \omega}\right)$ and a weighting filter $W\left(e^{i \omega}\right)$ emphasizing the low frequency region, which are shown in Figure 5. From the FRF data in Figure 4, it can be observed that locally, the system displays 2 nd order behavior. For this reason, a 2nd-order FIR controller structure is proposed. Furthermore, the basis functions $\left\{\phi_{j}^{\ell}(p)\right\}_{\ell=0, j=1}^{n_{\mathrm{b}}=2, n=2}$ are chosen to be affine functions of the operating point $p$, i.e., $\left\{\phi_{j}^{\ell}(p)\right\}_{j=1}^{n}=\{1, p\}, \forall \ell \in\{0,1,2\}$. To achieve zero steady-state error, integral action is enforced in the controller. This is achieved by (i) including an integrator in the plant FRFs, (ii) following the synthesis procedure described in Section III, and (iii) adding the integrator back to the controller. Note that this does not violate the assumptions.

First an LTI FIR controller is synthesized for a nominal operating point $p=0$, for which a magnitude plot is shown 

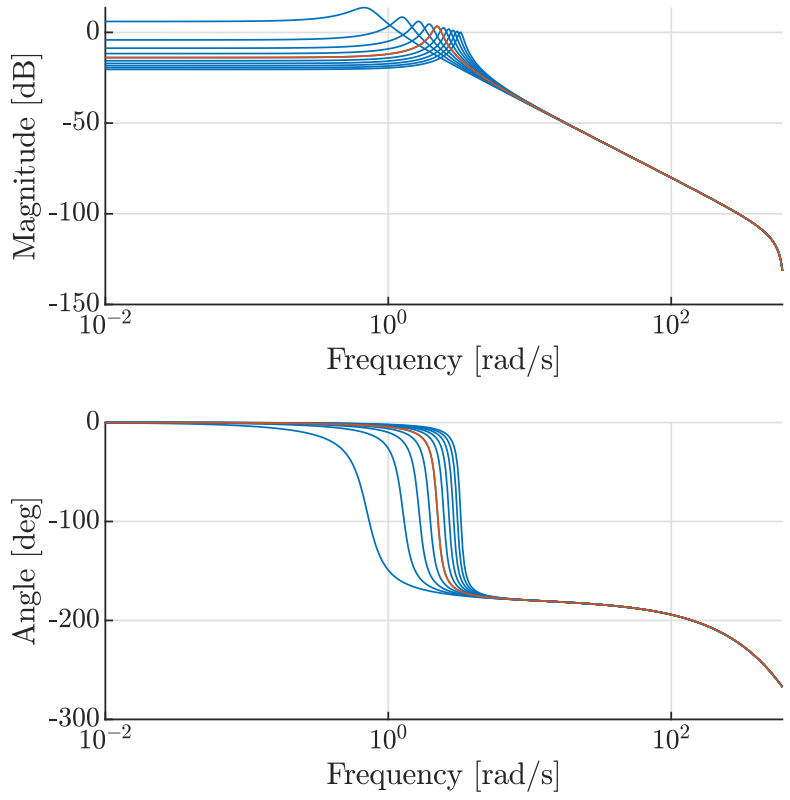

Fig. 4. Magnitude and phase plots of the frozen FRF data of the MSD system obtained at the operating points $\left\{p^{(\tau)}\right\}_{\tau=1}^{N_{p}} \subset[-1,1]$ are plotted in blue and the nominal FRF at $p=0$ is plotted in orange.

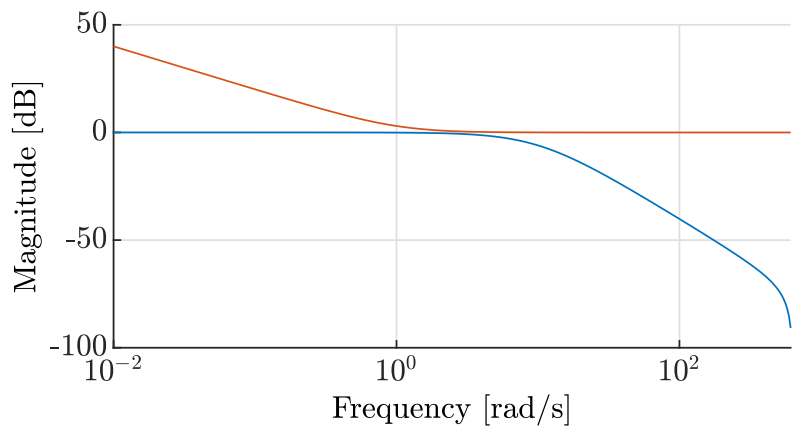

Fig. 5. Magnitude plot of the desired closed-loop behavior captured in terms of a model matching filter $M\left(e^{i \omega}\right)$ and the weighting filter $W\left(e^{i \omega}\right)$ emphasizing the low-frequency range, in blue and orange, respectively.

in Figure 7. The performance is assessed on a unit step response shown in Figure 6. This results in a stable closedloop with sufficient performance when the system is in the neighborhood of the operating point. However, a deviation from the operating point can lead to degradation in performance or even unstable behavior. Utilizing the proposed LPV approach, an LPV FIR controller is synthesized according to the presented methodology. A magnitude plot for frozen $p$ is shown in Figure 7. For comparison, also the magnitude plots of LTI controllers synthesized at $p=\{-1,1\}$ are shown. The LPV controller is able to adapt the controller coefficients based on the information of the scheduling variable and therefore significantly increases the achievable performance while maintaining local stability for the operating points $\mathcal{P}$, as shown in Figures 6 and 7.

Figure 8 shows the magnitude plot of the $T_{p}\left(e^{i \omega}\right)$ for a fine grid of $\mathbb{P}$. This shows that over the operating range $\mathbb{P}$,

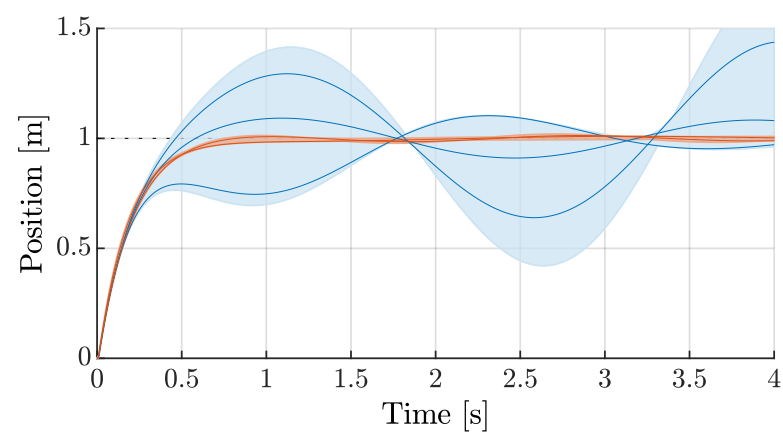

Fig. 6. Unit step response of the closed-loop system evaluated at randomly selected frozen operating points in $\mathbb{P}$, using the designed LTI and LPV FIR controllers in blue and orange, respectively. The shaded areas indicate the regions that cover trajectories for all frozen values in $\mathbb{P}$ for the LTI and LPV controllers in blue and orange, respectively. The performance of the LTI controller degrades for deviations from the operating point, whereas the LPV controller is able to maintain performance and stability

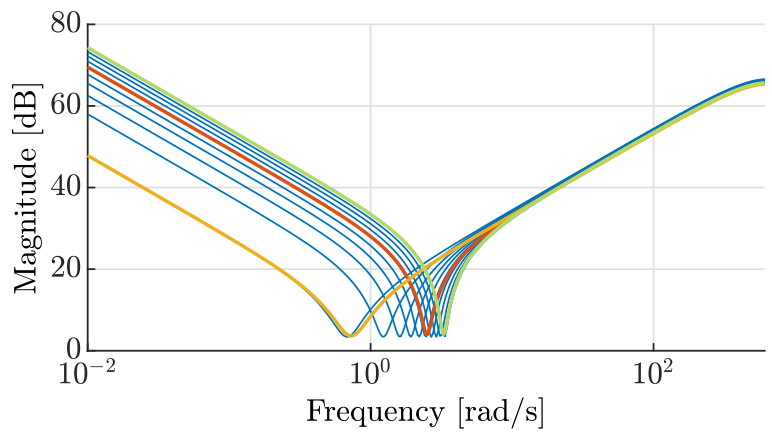

Fig. 7. Magnitude plot of the synthesized LPV and LTI controllers. The LPV controller is sampled on the grid $\mathcal{P}$, shown in blue. The LTI controllers correspond to the operating points $p=\{-1,0,1\}$, shown in yellow orange and green, respectively. The LPV controller adapts its coefficients to match, locally, the behavior of the LTI controllers at different operating points.

the variation with respect to $p$ is minimal, indicating that the performance specifications with respect to the model matching filter $M\left(e^{i \omega}\right)$ are met over the whole $\mathbb{P}$.

In Figure 9, the step response of the closed-loop nonlinear system with the synthesized 2nd-order discrete-time LPV FIR controller implemented according to Figure 2 is shown. Note that in this case, in (11) $p$ is time-varying, i.e.,

$$
u(k)=\sum_{\ell=0}^{n_{\mathrm{b}}=2}\left(c_{1}^{\ell}+c_{2}^{\ell} p(k)\right) e((k-\ell)),
$$

with $p(k)=\mu(x(k))=m_{c}+w_{c} x^{2}(k)$, where $k$ denotes discrete-time under a sampling period $T_{s}$. It can be observed that also stability for time-varying scheduling trajectories $p(k) \in \mathbb{P}$ is obtained, but a degradation in performance can be observed in comparison to the local results that are shown in Figure 6. In general, stability can only be guaranteed for parameters that vary sufficiently slow.

\section{CONCLUSIONS}

In this paper, a method is developed to directly synthesize a fixed-order LPV FIR controller based on measurement 


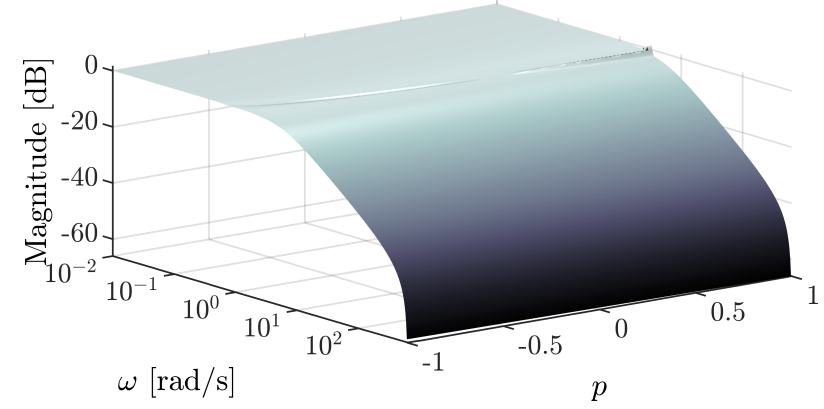

Fig. 8. Bode magnitude plot of the frozen frequency response of the closed-loop system at a fine grid of $\mathbb{P}$, indicating that the local variations w.r.t. $p$ are minimal

data. Through a global parametrization of the controller that is linear in the parameters, local stability and performance can be guaranteed with the proposed method. The presented method is not limited to FIR bases, but can be extended to any stable orthonormal basis functions. Through a simulation example it is shown that the synthesized LPV controller is able to adapt itself to the operating conditions of the system and therefore provides a significant increase in performance compared to LTI controllers. The method shows promising applications in areas where FRF measurements are utilized to design controllers, and the system behavior varies with operating conditions, for example, in the high-tech mechatronic industry. Future work includes the extension towards unstable systems and multi-input multi-output systems while aiming at a data-driven global stability concept.

\section{REFERENCES}

[1] J. M. Maciejowski, "Multivariable feedback design. electronic systems engineering series," Addison-Wesley, vol. 6, pp. 85-90, 1989.

[2] E. Grassi, K. S. Tsakalis, S. Dash, S. V. Gaikwad, W. MacArthur, and G. Stein, "Integrated system identification and PID controller tuning by frequency loop-shaping," IEEE Transactions on Control Systems Technology, vol. 9, no. 2, pp. 285-294, 2001.

[3] R. Hoogendijk, A. den Hamer, G. Angelis, R. van de Molengraft, and M. Steinbuch, "Frequency response data based optimal control using the data based symmetric root locus," in Proc. of the IEEE International Conference on Control Applications, Yokohama, Japan, October 2010, pp. 257-262.

[4] G. Galdos, A. Karimi, and R. Longchamp, "Robust loop shaping controller design for spectral models by quadratic programming," in Proc. of the 46th IEEE Conference on Decision and Control, New Orleans, USA, January 2007, pp. 171-176.

[5] A. Karimi and G. Galdos, "Fixed-order $\mathcal{H}_{\infty}$ controller design for nonparametric models by convex optimization," Automatica, vol. 46, no. 8, pp. 1388-1394, 2010.

[6] S. Khadraoui, H. Nounou, M. Nounou, A. Datta, and S. P. Bhattacharyya, "Robust control design method for uncertain system using a set of measurements," in Proc. of the American Control Conference, Washington, USA, August 2013, pp. 4325-4330.

[7] Y. Sakamoto, E. Nobuyama, and Y. Kami, "Robust controller design with fixed-order controllers using FNFR models," in Proc. of the 11th Asian Control Conference, Gold Coast, Australia, December 2017, pp. 1996-2000.

[8] H. Hjalmarsson, "From experiment design to closed-loop control," Automatica, vol. 41, no. 3, pp. 393-438, 2005.

[9] K. Zhou, J. C. Doyle, K. Glover et al., Robust and optimal control. Prentice hall, New Jersey, 1996, vol. 40.

[10] C. Scherer, "Mixed $\mathcal{H}_{2} / \mathcal{H}_{\infty}$ Control," in Trends in Control. Springer, London, 1995, pp. 173-216.

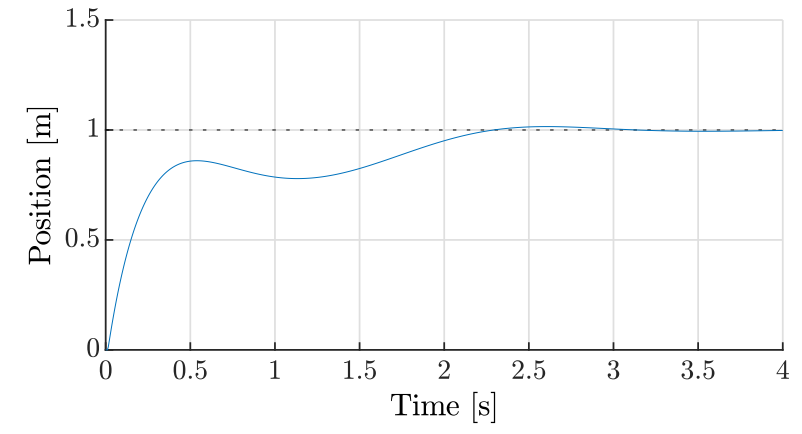

Fig. 9. Unit step response of the closed-loop nonlinear system using the 2nd order LPV FIR controller. A degradation in performance is observed for varying $p$.

[11] J. Mohammadpour and C. W. Scherer, Control of linear parameter varying systems with applications. Springer-Verlag, New York, 2012.

[12] M. G. Wassink, M. van de Wal, C. Scherer, and O. Bosgra, "LPV control for a wafer stage: beyond the theoretical solution," Control Engineering Practice, vol. 13, no. 2, pp. 231-245, 2005.

[13] C. Hoffmann and H. Werner, "A survey of linear parameter-varying control applications validated by experiments or high-fidelity simulations," IEEE Transactions on Control Systems Technology, vol. 23, no. 2, pp. 416-433, 2015.

[14] A. Packard, "Gain scheduling via linear fractional transformations," Systems \& control letters, vol. 22, no. 2, pp. 79-92, 1994.

[15] C. W. Scherer, "LPV control and full block multipliers," Automatica, vol. 37, no. 3, pp. 361-375, 2001.

[16] A. Hjartarson, P. Seiler, and A. Packard, "LPVTools: A toolbox for modeling, analysis, and synthesis of parameter varying control systems," in Proc. of the 1st IFAC Workshop on Linear Parameter Varying Systems, Grenoble, France, October 2015, pp. 139-145.

[17] A. van der Maas, R. van der Maas, R. Voorhoeve, and T. Oomen, "Frequency response function identification of LPV systems: a 2DLRM approach with application to a medical X-ray system," in Proc. of the American Control Conference, Boston, USA, July 2016, pp. 4598-4603.

[18] R. van der Maas, A. van der Maas, R. Voorhoeve, and T. Oomen, "Accurate FRF identification of LPV systems: nD-LPM with application to a medical X-ray system," IEEE Transactions on Control Systems Technology, vol. 25, no. 5, pp. 1724-1735, 2017.

[19] R. de Rozario and T. Oomen, "Frequency response function identification of LPV systems: a global approach with application to mechanical systems," in Proc. of the 18th IFAC Symposium on System Identification, Stockholm, Sweden, July 2018, pp. 108-113.

[20] M. Schoukens and R. Tóth, "Frequency response functions of linear parameter-varying systems," in Proc. of the 3rd IFAC Workshop on Linear Parameter Varying Systems, Eindhoven, the Netherlands, November 2019.

[21] M. Kunze, A. Karimi, and R. Longchamp, "Gain-scheduled controller design by linear programming," in Proc. of the European Control Conference, Kos, Greece, July 2007, pp. 5432-5438.

[22] A. Karimi and Z. Emedi, " $\mathcal{H}_{\infty}$ gain-scheduled controller design for rejection of time-varying narrow-band disturbances applied to a benchmark problem," European Journal of Control, vol. 19, no. 4, pp. 279-288, 2013.

[23] J. S. Shamma and M. Athans, "Gain scheduling: Potential hazards and possible remedies," IEEE Control Systems Magazine, vol. 12, no. 3, pp. 101-107, 1992.

[24] R. Tóth, Modeling and identification of linear parameter-varying systems. Springer, Berlin Heidelberg, 2010.

[25] S. Skogestad and I. Postlethwaite, Multivariable feedback control: analysis and design. Wiley, New York, 2007, vol. 2.

[26] D. Vizer and G. Mercere, " $\mathcal{H}_{\infty}$-based LPV model identification from local experiments with a gap metric-based operating point selection," in Proc. of the European Control Conference, Strasbourg, France, June 2014, pp. 388-393. 